\title{
INTERFERÊNCIA DA DISFAGIA OROFARÍNGEA NO CONSUMO ALIMENTAR DE INDIVÍDUOS COM MUCOPOLISSACARIDOSE II
}

\author{
Interference of oropharyngeal dysphagia in food consumption \\ in patients with mucopolysaccharidosis II
}

\author{
Ana Carolina Rocha Gomes Ferreira ${ }^{(1)}$, Alane Cabral de Oliveira ${ }^{(2)}$, Larissa de Lima Pessoa Veiga ${ }^{(3)}$, \\ Liziane Damasceno Santana ${ }^{(4)}$, Pauliana Buarque Barbosa ${ }^{(5)}$, Zelita Caldeira Ferreira Guedes ${ }^{(6)}$
}

\begin{abstract}
RESUMO
Objetivo: o presente estudo visou relacionar o grau de disfagia com o consumo alimentar de indivíduos com mucopolissacaridose II (MPS II). Método: foram incluídos indivíduos com MPS II do departamento de genética da Universidade Estadual de Alagoas e excluídos aqueles com outros tipos de mucopolissacaridoses, bem como que estivessem em uso de via alternativa de alimentação. Realizadas avaliações antropométrica, dietética, fonoaudiológica para disfagia, clínica otorrinolaringológica e a videoendoscopia da deglutição. Resultados: foram estudados 07 indivíduos, do gênero masculino, entre 5 e 14 anos de idade, dos quais mais de $50 \%$ faziam uso de anti-hipertensivo e $42,8 \%$ manifestavam a forma grave da doença. Seis deles apresentaram déficit de altura/ idade e mais de $70 \%$ encontravam-se obesos segundo o Índice de Massa Corporal (IMC). Foi observada disfagia em cinco deles, com média diária de consumo calórico de 920,15 $\pm 244,09$ calorias, contra 1264,94 \pm 106,85 calorias para aqueles sem disfagia, com variação intra-individual significativamente maior no grupo de portadores de disfagia $(p<0,05)$. Além disso, os indivíduos sem disfagia apresentaram consumo alimentar mais elevado de carboidratos, proteínas e lipídios. Já para os micronutrientes, com exceção da média de consumo diária de ferro e vitamina $\mathrm{E}$, todos os outros avaliados apresentaram médias diárias de consumo maiores no grupo sem disfagia $(p<0,05)$. Conclusão: foi observada uma elevada frequência de disfagia nos portadores de MPS II estudados, e isso foi associado ao baixo consumo alimentar calórico e desequilíbrio na proporção e quantidade de macronutrientes e de parte dos micronutrientes.
\end{abstract}

DESCRITORES: Transtornos da Deglutição; Mucopolissacaridose II; Dieta; Consumo de Alimentos

(1) Fonoaudióloga; Professora Auxiliar da Faculdade de Fonoaudiologia de Alagoas - UNCISAL; Maceió, Alagoas, Brasil; Mestre em Distúrbio da Comunicação Humana pela Universidade Federal de São Paulo - UNIFESP; Especialização em Motricidade Orofacial pela UFPE;

(2) Nutricionista; Professora Adjunto da Universidade Federal de Alagoas; Maceió, Alagoas, Brasil.

(3) Nutricionista; Graduada pela Escola de Ciências da Saúde do Centro de Estudos Superiores de Maceió - CESMAC; Maceió, Alagoas, Brasil.

(4) Nutricionista; Graduada pela Escola de Ciências da Saúde do Centro de Estudos Superiores de Maceió - CESMAC; Maceió, Alagoas, Brasil.

(5) Nutricionista; Graduada pela Escola de Ciências da Saúde do Centro de Estudos Superiores de Maceió - CESMAC; Maceió, Alagoas, Brasil.

(6) Fonoaudióloga; Professora Associado II da Universidade Federal de São Paulo - UNIFESP, São Paulo, São Paulo, Brasil; Doutora em Distúrbio da Comunicação Humana pela Universidade Federal de São Paulo - UNIFESP.

Conflito de interesses: inexistente

\section{INTRODUÇÃO}

As mucopolissacaridoses (MPS) fazem parte de um grupo de doenças metabólicas hereditárias causadas por erros inatos do metabolismo dos glicosaminoglicanos (GAGs) ${ }^{1,2}$. Esta doença é conseqüência da deficiência de determinadas enzimas responsáveis pela degradação dos GAGs, resultando no acúmulo de tais substratos dentro dos lisossomos ${ }^{1-3}$.

O padrão de herança das MPS é autossômico recessivo, com exceção da mucopolissacaridose II (MPS II), que é ligada ao cromossomo X. A MPS II, também chamada de Síndrome de Hunter, pode ser classificada como leve ou grave, de acordo com o comprometimento do sistema nervoso central ${ }^{3-5}$. 
As manifestações clínicas nas MPS são variadas, a exemplo: comprometimento respiratório; cardiopatias; alterações neurológicas, dentre outras ${ }^{2-4}$. Além disso, são descritas na literatura alterações nas funções orais que repercute negativamente sobre o estado nutricional5 ${ }^{5,6}$.

Os distúrbios nutricionais, que também fazem parte do quadro de sintomas da MPS II são decorrentes de disfunções gastrintestinais como a diarréia crônica, constipação, alterações na mastigação e principalmente a disfagia orofaríngea. Esta última pode causar um impacto nutricional importante em virtude da diminuição da ingestão alimentar ${ }^{6}$.

Em pacientes disfágicos são comuns as queixas de recusa alimentar, dificuldade de iniciar a deglutição, irritação freqüente na faringe com necessidade de "limpeza", regurgitação nasal, escape oral, controle da produção de saliva alterado, tosse e/ ou engasgo durante as refeições. Outro cuidado importante em relação ao paciente disfágico está relacionado ao risco de aspiração, como também, ao perigo da desidratação e desnutrição ${ }^{7}$.

$\mathrm{Na}$ literatura pesquisada foi encontrado um estudo de caso de um indivíduo com MPS II do tipo grave, em estágio avançado da doença, com disfagia orofaríngea grave e importante comprometimento na linguagem compreensiva ${ }^{8}$. Os indivíduos disfágicos, sobretudo com comprometimento neurológico, podem comprometer a proteção das vias aéreas e perder a possibilidade de nutrição adequada ${ }^{7}$.

Em outro estudo foram avaliadas vinte e oito crianças com MPS, em que se observaram quadros de disfagia nas fases preparatória, oral, e faríngea. Das vinte e oito crianças, oito apresentaram casos graves de pneumonias aspirativas com indicação de via alternativa de alimentação?

Portanto, a MPS II é uma doença de caráter progressivo, com manifestações clínicas agressivas que afetam diversas partes do corpo, inclusive 0 trato gastrointestinal, comprometendo frequentemente o estado nutricional de seus portadores. Além disso, não existem estudos descritos na literatura que abordem questões relacionadas $s$ à influência da disfagia sobre a alimentação neste público alvo. Assim, o presente estudo é justificado, e tem como objetivo relacionar o grau de disfagia com o consumo alimentar de indivíduos com MPS II.

\section{MÉTODO}

Esse estudo é do tipo transversal realizado com portadores de MPS II do estado de Alagoas.

A presente pesquisa foi realizada na Unidade de Tratamento em Fonoaudiologia Professor Jurandir
Bóia Rocha na Universidade Estadual de Ciências da Saúde de Alagoas - UNCISAL.

Foram incluídos no estudo todos os indivíduos diagnosticados clinicamente e bioquimicamente com MPS II e registrados no departamento de genética da UNCISAL, que é referência nesta patologia e engloba toda a população assistida no estado de Alagoas. Foram excluídos aqueles com outros tipos de MPS, indivíduos que estivessem fazendo uso de via alternativa de alimentação (sonda enteral ou parenteral) e aqueles que estivessem em tratamento de reposição enzimática específico para MPS.

Inicialmente foi aplicado um questionário padronizado referente as características clínicas e demográficas do grupo estudado. Após, foram realizadas as seguintes avaliações: antropométrica ${ }^{10}$, dietética ${ }^{11}$, fonoaudiológica para disfagia ${ }^{12}$, avaliação clínica otorrinolarigológica e a videoendoscopia da deglutição (VED) ${ }^{13}$.

A avaliação antropométrica foi realizada seguindo recomendações estabelecidas pela Organização Mundial da Saúde ${ }^{10}$, a partir da coleta de dados referentes a peso e estatura. Assim, os indivíduos foram pesados descalços e com roupas leves, posicionados de costas para a balança, no centro do equipamento, ereto, com os pés juntos e os braços estendidos ao longo do corpo mantido parado nessa posição. Foi utilizada uma balança antropométrica mecânica de marca FILIZOLA®, com capacidade de $150 \mathrm{~kg}$, com precisão de $100 \mathrm{~g}$ e previamente calibrada. Para aferição da estatura, foi utilizado estadiômetro da própria balança (que foi aferido o peso). A aferição da estatura foi realizada em centímetros.

Em posse dos dados de peso e altura foram calculados o IMC (Índice de Massa Corpórea) e os indicadores antropométricos de altura para idade $(\mathrm{A} / \mathrm{I})$, peso para altura $(\mathrm{P} / \mathrm{A})$ e peso para idade $(\mathrm{P} / \mathrm{I})$ de cada indivíduo.

A avaliação dietética ${ }^{11}$ foi feita a partir do inquérito dietético, recordatório de 24 horas, a fim de avaliar o consumo alimentar, bem como, estimar quantitativamente e qualitativamente a ingestão dos alimentos consumidos pelos indivíduos para detectar possíveis fatores de risco para carências dietéticas específicas, hábitos alimentares, além de tabus, preferências e alergias alimentares. O recordatório de 24 horas consiste no registro de informações de todos os alimentos consumidos e suas porções ingeridas nas últimas 24 horas. Foram realizados em cinco dias alternados (24horas, $3,8,15$ e 30 dias) a fim de minimizar erros refletidos de um dia atípico. Este foi realizado mediante entrevista pessoal individualizada com as mães dos 
indivíduos já que os mesmos não estavam aptos a uma descrição correta, seja pela idade, ou pela MPS.

Os macronutrientes e micronutrientes foram avaliados de acordo com as Recomendações Diárias de Ingestão (DRIs) ${ }^{14}$. Já as necessidades energéticas dos indivíduos foram calculadas segundo o Instituto de Medicina (IOM, 2002/ $2005)^{15}$. Para o cálculo das necessidades energéticas foram consideradas a idade, peso, estatura, bem como o fator atividade. Foi considerado o fator atividade de 1,3 , correspondente a atividade muito leve ou sedentarismo, devido a doença que limita as atividades diárias de seus portadores. Os indivíduos em estudo foram estratificados segundo as faixas etária de 4-8 anos, 9-13 anos e 14-18 anos, visto as DRIs serem específicas por faixas etárias.

A avaliação fonoaudiológica ${ }^{12}$ escolhida apresenta características clínicas e fonoaudiológicas semelhantes à população estudada. Para realização desta avaliação foram utilizados os seguintes materiais: luvas, espátulas, alimentos de consistência líquida (água filtrada à temperatura ambiente), pastosa (iogurte) e sólida (pão tipo francês de 25 gramas), além do estetoscópio pediátrico da marca Littmann.

Nesta avaliação foi seguido o protocolo que avalia os seguintes aspectos: lábios ocluídos; elevação laríngea; protrusão de língua durante a captação do bolo; tempo de trânsito oral; sustentação da mandíbula durante a deglutição; presença ou ausência de tosse antes, durante ou após a deglutição; presença ou ausência de regurgitação nasal; qualidade vocal com característica seca ou molhada; resíduo alimentar após a deglutição; presença ou ausência de escape extra oral de alimentos; ausculta cervical se apresenta sinais sugestivos de aspiração ou penetração. Para a ausculta cervical foi colocado o diafragma do estetoscópio no bordo lateral da traquéia, imediatamente inferior a cartilagem cricóide. Foi classificada como deglutição normal o ruído caracterizado por dois cliques seguido do ruído expiratório. Os ruídos fora deste padrão foram considerados ausculta cervical alterada. Quanto à mandíbula, considerou-se boa estabilidade, quando os dentes encontravam-se em oclusão cêntrica, ou sem estabilidade quando as arcadas dentárias encontrava-se entreabertas.

As avaliações clínica otorrinolarigológica e a videoendoscopia da deglutição (VED) ${ }^{13}$ foram feitas segundo o protocolo da Faculdade de Fonoaudiologia da UNCISAL. A avaliação foi dividida em duas etapas e realizada por um otorrinolaringologista acompanhado por uma fonoaudióloga. Os indivíduos foram colocados sentados no colo do acompanhante e foi explanado sobre a realização do exame, quando não colaboravam os mesmos foram contidos para evitar movimentos espontâneos, ou reflexos que viessem a interferir o exame. Os resultados foram classificados segundo a escala de comprometimento funcional (gravidade) da deglutição. Esta é a escala preconizada por Macedo Filho (2003) $)^{13}$ que classifica os achados da avaliação videoendoscópica da deglutição. Nesta etapa do processo avaliativo analisa indiretamente a fase oral, por meio da capacidade de controlar o alimento na boca, e se ocorre escape de alimentos para a valécula e seios piriformes antes da deglutição, com ou sem aspiração. Também pode-se observar neste procedimento o grau de elevação e fechamento do palato, assim como a coordenação de sua elevação com a constrição faríngea preparatórios para a fase seguinte.

A partir desta escala a disfagia pode ser classificada em: grau zero (normal), ausência de alterações; grau I, estase pós-deglutição, menos de três tentativas para clareamento, ausência de regurgitação nasal, e penetração laríngea; grau 2 (moderada) estase salivar moderada, maior estase pós deglutição, mais de três tentativas de propulsão do bolo, regurgitação nasal, redução da sensibilidade laríngea com penetração, porém sem aspiração laringotraqueal e grau 3 (severa) grande estase salivar, piora acentuada de resíduos pós deglutição, propulsão débil ou ausente, regurgitação nasal, aspiração traqueal.

A avaliação estrutural e funcional da deglutição ${ }^{13}$ teve por finalidade verificar os aspectos estruturais pela avaliação das pregas vocais, laringe, hipofaringe, orofaringe, cavidade oral e porção superior da traqueia e funcionalidade da deglutição. Este exame é realizado por meio de uma aparelhagem de fibra ótica, rígida ou flexível, conectada a uma micro-câmera e um vídeo cassete. No teste da deglutição utilizando a mesma aparelhagem, não é possível verificar a fase oral, entretanto foi obtido informações úteis quanto a capacidade de contenção dos alimentos, escape precoce da fase oral, resíduo faríngeo, penetração laríngea, aspiração laríngea ou regurgitação esofágica ${ }^{12}$.

Primeiramente, foram avaliados os aspectos estrutural e funcional da deglutição, sem a utilização de alimento, sendo observadas as fossas nasais, rinofaringe e esfíncter velofaríngeo durante a deglutição espontânea de saliva. Por deflexão inferior do aparelho foram observadas as seguintes estruturas: a base da língua, valéculas, parede lateral e posterior da faringe, recessos piriformes, laringe e a presença de estase salivar nestas estruturas. A mobilidade e motilidade das pregas vocais foram observadas durante o choro e/ou deglutição espontânea de saliva, durante a fala quando o 
indivíduo apresentava a habilidade de fonação ou de responder às ordens simples para verbalizar vogais e palavras ${ }^{13}$. Na segunda etapa foi realizada a avaliação funcional da deglutição propriamente dita, oferecendo alimentos corados com azul de anilina em quantidades e consistências progressivas de acordo com as possibilidades de cada indivíduo. Foi avaliada também a sensibilidade faringolaríngea e a identificação de ocorrência ou não de penetração e aspiração de secreções ou alimentos. Para o equivalente à consistência líquida, foi oferecida água filtrada à temperatura ambiente, iogurte correspondendo à consistência pastosa e pão do tipo francês $(25 \mathrm{~g})$ representando a consistência sólida.

Nas avaliações otorrinolarigológica, estrutural e funcional da deglutição foi utilizado endoscópio convencional composto por: aparelho televisor SEMP®, 10 polegadas, colorido, modelo TV 1022FAVU11; vídeo cassete SEMP®, Hi-Fi stéreo sete cabeças VHS-HQ; micro-câmara Toshiba ${ }^{\circ}$ A43, acoplada a um adaptador para endoscópio Machida $\AA$, modelo CA-34VS2; fibronasofaringolaringoscópio flexível Olympus $₫$ 4,2 mm ENF tipo 10; fonte de luz halógena Olympus $₫$ CLE - F10 com flash e fonte metal hilide Welc Allyn $\AA$. Os exames foram transcritos em DVD, permitindo revisões de imagem. $O$ resultado da avaliação instrumental foi classificado de acordo com grau de comprometimento funcional da deglutição ${ }^{13}$.

O presente estudo foi aprovado pelo Comitê de Ética e Pesquisa da Universidade Estadual de Ciências da Saúde de Alagoas com número de protocolo 724 . Os responsáveis pelos indivíduos foram avaliados segundo TCLE (Termo de Consentimento Livre e Esclarecido).

A análise dos dados foi realizada com o auxílio do programa SPSS versão 16.0 e o nível de significância utilizado foi de $5 \%$. Foram utilizados os testes $\mathrm{T}$, qui-quadrado e de correlação bivariada, sendo este último com o grau de relacionamento linear avaliado pelo coeficiente de Spearman. $O$ programa Virtual Nutri 1.0, foi utilizado para avaliar quantitativamente o consumo alimentar, e as DRl's $(2001)^{14}$ serviram de parâmetro para avaliar o índice de adequação de ingestão alimentar de cada paciente.

\section{RESULTADOS}

Foram estudados 07 indivíduos com MPS II, de um total de 14 cadastrados no estado de Alagoas, do gênero masculino, na faixa etária de 5 a 14 anos de idade. Pelos critérios de inclusão e exclusão totalizou-se $50 \%$ da amostra de todo o estado. Dentre os indivíduos estudados, apenas 01 nasceu prematuramente. As famílias desses menores podem ser consideradas de baixa renda, visto que apresentavam uma renda mensal menor que 1 salário mínimo. Porém, todos recebiam ajuda do governo. As mães dos mesmos eram de baixa escolaridade ou analfabetas. Além disso, mais de $50 \%$ deles faziam uso de anti-hipertensivo, $42,8 \%$ manifestavam a forma severa da doença e nenhum deles fazia uso de enzimas repositoras ou estavam em terapia nutricional (sonda enteral ou parenteral). As características demográficas e clínicas da população estudada podem ser vista na Tabela 1.

O estado nutricional dos portadores de MPSII foi avaliado segundo o IMC e indicadores antropométricos de peso para a altura (P/A), peso para idade $(P / I)$ e altura para idade $(A / I)$, como pode ser observado nas figuras 1 e 2, respectivamente. Assim, $85,71 \%(n=6)$ dos portadores de MPS II apresentaram déficit de $\mathrm{A} / \mathrm{I}$, destes três com déficit grave, o que é característico de uma desnutrição crônica ou pregressa. Déficit de P/I foi observado em $71,42 \%$ dos portadores de MPS II, comum na desnutrição aguda. Em contrapartida, cinco deles encontravam-se com excesso de peso segundo o IMC.

A Figura 3 mostra o consumo calórico versus necessidades energéticas dos indivíduos estudados. Foram encontradas médias calóricas de $1004,38 \pm 270,76$ calorias e $1360,1 \pm 126,69$ calorias $(p<0,05)$ de consumo alimentar e necessidades energéticas diárias, respectivamente. Além disso, ao estratificar a amostra entre aqueles com a forma leve e severa da doença (dano neurológico) foram observadas médias diárias de consumo calórico de: $1213,71 \pm 88,24$ calorias e 725,27 $\pm 61,21$ calorias $(p<0,001)$ para as formas leve e severa, respectivamente.

$\mathrm{Na}$ Tabela 2 pode ser visto o consumo alimentar de macronutrientes e micronutrientes por faixa etária nos portadores de MPS II. Assim, os indivíduos estudados apresentaram médias de consumo diário elevadas de carboidratos, baixo consumo diário de proteínas nas faixas etárias de 9-13 anos e 14-18 anos, e elevado consumo de lipídios para a faixa etária de 4-8 anos. Já em relação ao consumo alimentar dos micronutrientes, foi observado um baixo consumo diário de cálcio, potássio, sódio e vitamina $\mathrm{E}$ em todos os portadores de MPS II estudados. Consumo aquém de ferro na faixa etária de 9-13 anos; de fósforo e zinco abaixo das recomendações para aqueles com mais de 9 anos de idade. Em contrapartida, foi observado um consumo adequado das vitaminas $\mathrm{A}$ e $\mathrm{C}$ em todas as faixas etárias. 
Tabela 1 - Características demográficas e clínicas de portadores de MPS II

\begin{tabular}{|c|c|c|}
\hline Características demográficas e clínicas & $\begin{array}{l}\text { Quantidade } \\
\text { (n) }\end{array}$ & $\begin{array}{c}\text { Porcentagem } \\
(\%)\end{array}$ \\
\hline \multicolumn{3}{|l|}{ Renda familiar } \\
\hline$<1$ salário mínimo & 7 & 100 \\
\hline >1 salário mínimo & 0 & 0 \\
\hline \multicolumn{3}{|l|}{ Recebimento de ajuda do governo } \\
\hline Sim & 7 & 100 \\
\hline Não & 0 & 0 \\
\hline \multicolumn{3}{|l|}{ Procedência } \\
\hline Capital (Maceió) & 1 & 14,28 \\
\hline Interior & 6 & 85,71 \\
\hline \multicolumn{3}{|l|}{ Escolaridade materna } \\
\hline Analfabetismo & $1^{*}$ & 25 \\
\hline Ensino fundamental & 3 & 75 \\
\hline \multicolumn{3}{|l|}{ Presença de Hipertensão Arterial Sistêmica } \\
\hline Sim & 5 & 71,43 \\
\hline Não & 2 & 28,57 \\
\hline \multicolumn{3}{|l|}{ Doença } \\
\hline Forma leve & 3 & 42,80 \\
\hline Forma severa & 4 & 57,20 \\
\hline \multicolumn{3}{|l|}{ Uso do enzimas repositoras } \\
\hline Sim & 0 & 0 \\
\hline Não & 7 & 100 \\
\hline \multicolumn{3}{|l|}{ Terapia nutricional } \\
\hline Sim & 0 & 0 \\
\hline Não & 7 & 100 \\
\hline
\end{tabular}

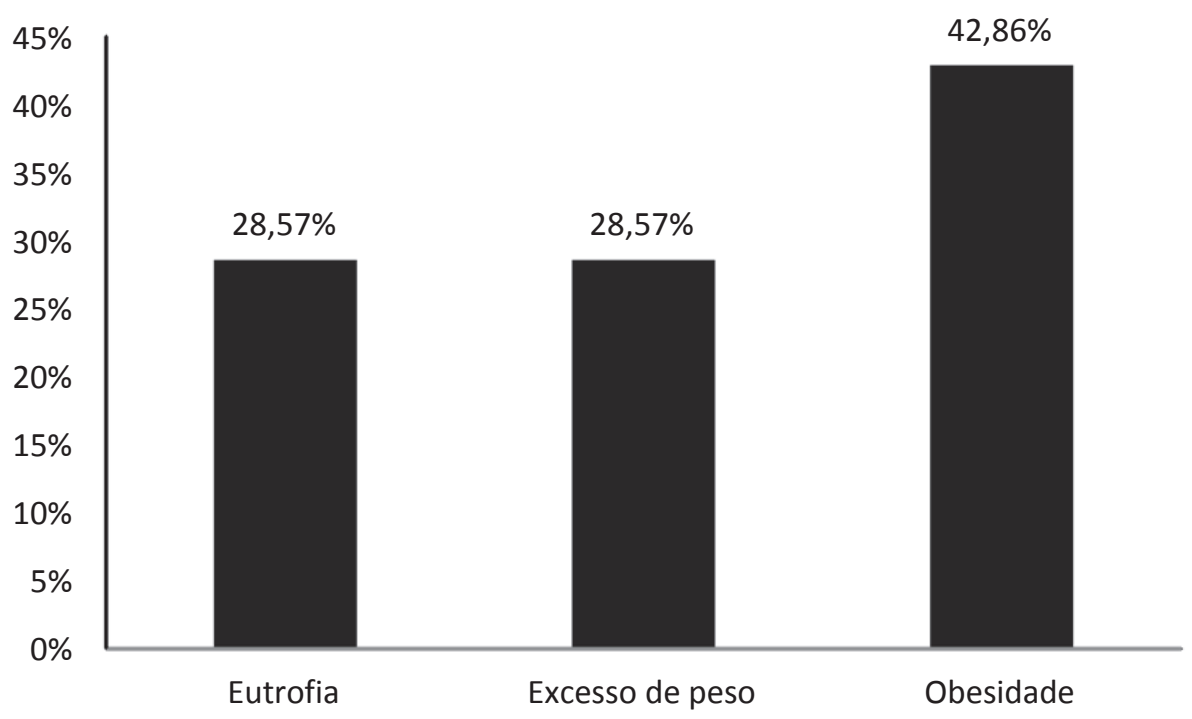

Figura 1 - Estado nutricional, segundo Índice de Massa Corporal (IMC), dos indivíduos com MPS II 


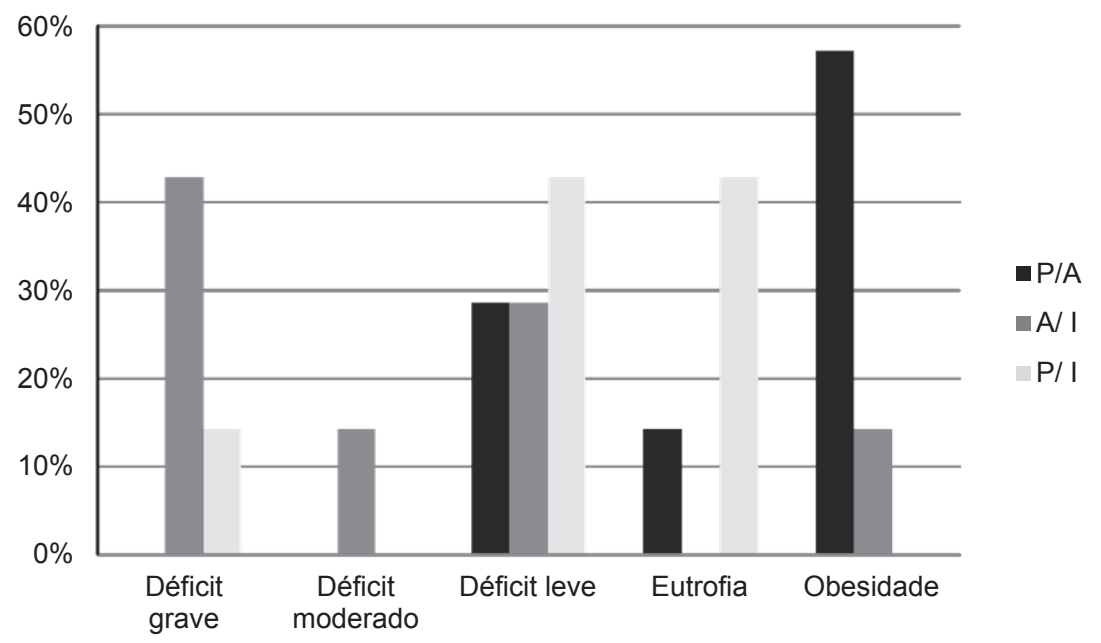

Figura 2 - Estado nutricional, segundo indicadores antropométricos de peso para a altura (P/A), peso para idade $(\mathrm{P} / \mathrm{I})$ e altura para idade $(\mathrm{A} / \mathrm{I})$

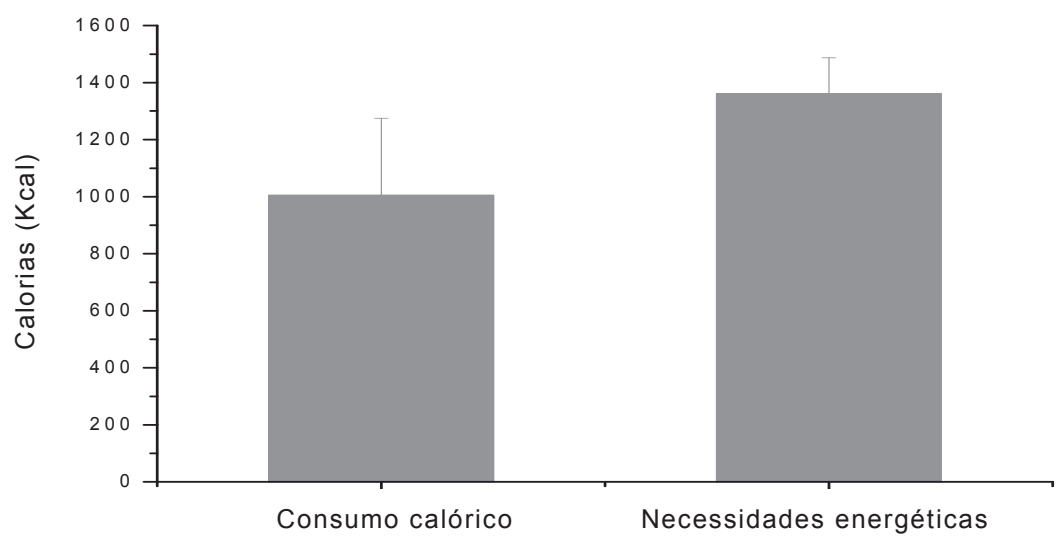

Figura 3 - Consumo calórico versus necessidades energéticas de indivíduos com MPS II

Tabela 2 - Média de consumo alimentar diário de macronutrientes e micronutrientes segundo faixa etária de portadores de MPS II

\begin{tabular}{lccc}
\hline \multicolumn{4}{c}{ Média diária de consumo de nutrientes } \\
\hline Nutrientes & $\mathbf{4 - 8}$ anos & $\mathbf{9 - 1 3}$ anos & $\mathbf{1 4 - 1 8}$ anos \\
& $\mathbf{n}=\mathbf{3}$ & $\mathbf{n}=\mathbf{3}$ & $\mathbf{n}=\mathbf{1}$ \\
\hline Carboidratos $(\mathrm{g})$ & $158,26 \pm 34,15$ & $142,04 \pm 30,63$ & $154,79 \pm 60,89$ \\
Proteínas $(\mathrm{g})$ & $36,94 \pm 23,19$ & $55,05 \pm 11,46$ & $11,2 \pm 3,15$ \\
Lipídios $(\mathrm{g})$ & $39,04 \pm 32,73$ & $26,17 \pm 5,71$ & $12,99 \pm 3,83$ \\
Cálcio $(\mathrm{mg})$ & $258,62 \pm 146,52$ & $128,74 \pm 49,36$ & $734,64 \pm 527,04$ \\
Ferro $(\mathrm{mg})$ & $9,6 \pm 1,39$ & $7,95 \pm 1,99$ & $20,50 \pm 22,70$ \\
Zinco $(\mathrm{mg})$ & $5,46 \pm 2,3$ & $4,90 \pm 1,40$ & $0,95 \pm 0,25$ \\
Sódio* $(\mathrm{mg})$ & $974,36 \pm 593,01$ & $902,05 \pm 403,53$ & $172,72 \pm 54,56$ \\
Potássio $(\mathrm{mg})$ & $1096,00 \pm 346,90$ & $528,17 \pm 429,42$ & $774,69 \pm 178,10$ \\
Fósforo $(\mathrm{mg})$ & $493,09 \pm 148,39$ & $407,28 \pm 37,18$ & $498,24 \pm 191,66$ \\
Vitamina C $(\mathrm{mg})$ & $109,15 \pm 42,09$ & $46,26 \pm 30,97$ & $115,77 \pm 86,86$ \\
Vitamina A $(\square \mathrm{g})$ & $1331,16 \pm 512,37$ & $1287,22 \pm 1069,70$ & $1111,48 \pm 999,94$ \\
Vitamina E $(\mathrm{mg})$ & $5,80 \pm 1,32$ & $4,76 \pm 0,06$ & $2,37 \pm 1,18$ \\
\hline
\end{tabular}


A escala de comprometimento funcional da deglutição demonstrou a presença de disfagia grau I (leve) em 42,85\% $(n=3)$ dos indivíduos, disfagia grau III (severa) em $28,57 \%(n=2)$ dos indivíduos, e $28,57 \%(n=2)$ não apresentaram tal alteração. Todos os indivíduos com a forma grave da doença $(n=3)$ apresentaram algum grau de disfagia.

Quando estratificados em relação à presença ou ausência de disfagia, a média de consumo calórico foi de 920,15 $\pm 244,09$ calorias/ dia para os indivíduos com algum grau de disfagia e de $1264,94 \pm 106,85$ calorias/dia para aqueles sem tal alteração $(p<0,05)$. Além disso, a ingestão de energia apresentou uma variação intra-individual dos cinco dias de registros alimentares de $10,17 \%$ a $30,33 \%$ no grupo de portadores de MPS II com disfagia, e de $12,74 \%$ a $19,55 \%$ no grupo sem disfagia, sendo significativamente maior no primeiro grupo $(p<0,05)$.

A Figura 4 mostra a relação entre o grau de disfagia dos indivíduos estudados e o consumo calórico diário. Como pode ser visto, houve associação negativa entre estes aspectos $(r=-0,82375)$, o que mostra que quanto maior o grau de disfagia, menor o consumo calórico diário.

Já na Tabela 3 encontra-se o consumo diário de ingestão de nutrientes nos portadores de MPS II com e sem disfagia. O grupo de indivíduos sem disfagia apresentou média de consumo alimentar mais elevada de carboidratos, de proteínas e lipídios, quando comparado com o grupo com disfagia. Já para os micronutrientes, com exceção da média de consumo diária de ferro e vitamina $\mathrm{E}$, todos os outros avaliados apresentaram médias diárias de consumo mais elevadas no grupo sem disfagia $(p<0,05)$.

A variabilidade do consumo dos micronutrientes na dieta desses indivíduos também foi avaliada segundo os grupos de indivíduos com e sem disfagia (Tabela 4). Assim, a variação na ingestão de todos os minerais estudados foi significativamente maior no grupo com disfagia $(p<0,05)$.

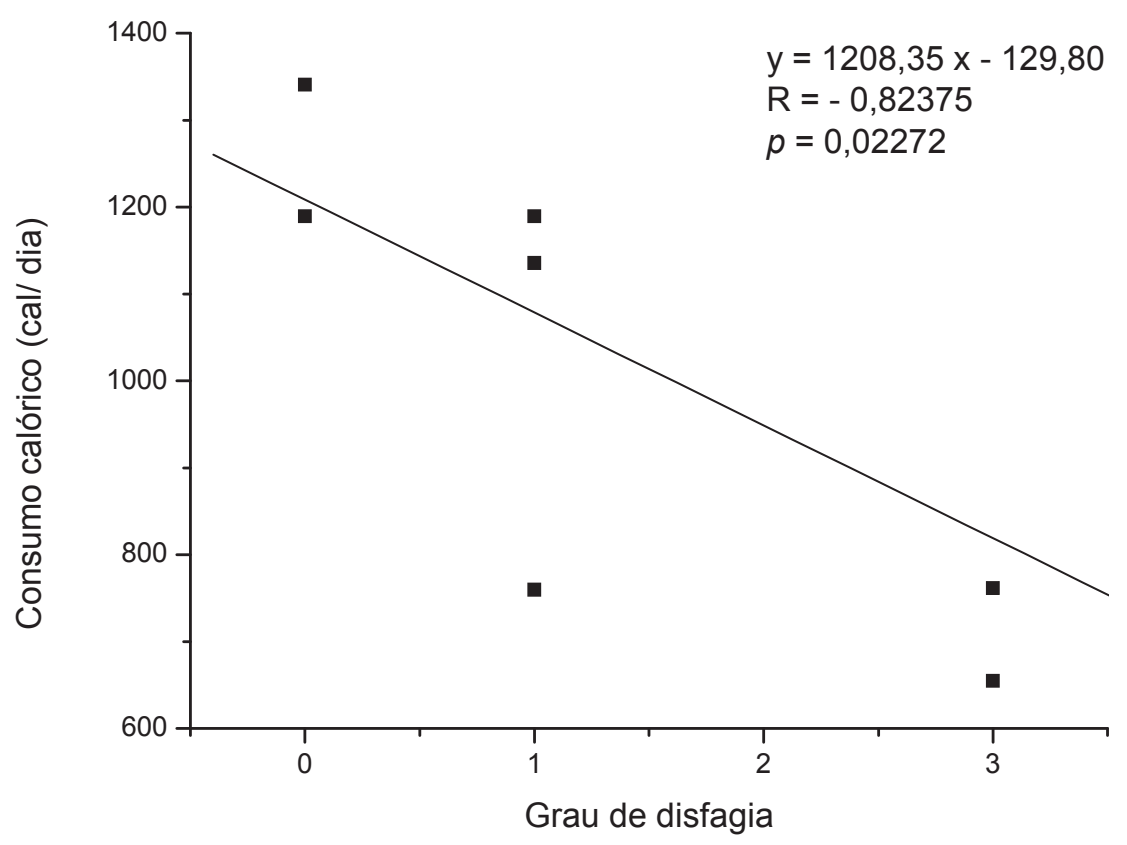

Figura 4 - Grau de disfagia versus consumo alimentar calórico (cal/dia) de portadores de MPSII 
Tabela 3 - Média de consumo alimentar diário de macronutrientes e micronutrientes dos indivíduos com MPS II, com e sem disfagia

\begin{tabular}{lccc}
\hline \multicolumn{3}{c}{ Média do consumo diário de nutrientes } \\
Nutrientes & $\begin{array}{c}\text { Indivíduos com } \\
\text { disfagia } \\
\mathbf{n}=\mathbf{5}\end{array}$ & $\begin{array}{c}\text { Indivíduos sem } \\
\text { disfagia } \\
\mathbf{n = 2}\end{array}$ & Valor de $\boldsymbol{p}^{* *}$ \\
\hline Carboidrato $(\mathrm{g})$ & $140,42 \pm 24,80$ & $176,79 \pm 16,46$ & $<0,05$ \\
Proteína $(\mathrm{g})$ & $23,48 \pm 14,46$ & $30,19 \pm 0,98$ & $<0,05$ \\
Lipídio $(\mathrm{g})$ & $34,66 \pm 24,79$ & $68,86 \pm 8,89$ & $<0,05$ \\
Cálcio $(\mathrm{mg})$ & $309,70 \pm 272,76$ & $174,09 \pm 9,14$ & $<0,05$ \\
Ferro $(\mathrm{mg})$ & $10,55 \pm 5,78$ & $10,20 \pm 1,18$ & 0,6743 \\
Zinco $(\mathrm{mg})$ & $3,71 \pm 2,02$ & $6,72 \pm 1,35$ & $<0,05$ \\
Sódio* $(\mathrm{mg})$ & $640,12 \pm 462,58$ & $1309,81 \pm 167,93$ & $<0,05$ \\
Potássio $(\mathrm{mg})$ & $810,29 \pm 178,26$ & $1229,21 \pm 368,46$ & $<0,05$ \\
Fósforo $(\mathrm{mg})$ & $421,46 \pm 51,07$ & $545,74 \pm 164,66$ & $<0,05$ \\
Vitamina C $(\mathrm{mg})$ & $74,06 \pm 43,91$ & $105,86 \pm 58,98$ & $<0,05$ \\
Vitamina A $(\square \mathrm{g})$ & $1228,50 \pm 760,90$ & $1411,90 \pm 697,09$ & 0,0832 \\
Vitamina E $(\mathrm{mg})$ & $4,38 \pm 1,14$ & $6,10 \pm 1,78$ & \\
\hline
\end{tabular}

*intrínsico

${ }^{* *}$ Teste $t$

Tabela 4 - Coeficiente de variabilidade entre portadores de MPS II com e sem disfagia

\begin{tabular}{lccc}
\hline \multicolumn{4}{c}{ Coeficiente de variabilidade (\%) } \\
\hline Micronutrientes & $\begin{array}{c}\text { Com disfagia } \\
\mathbf{n = 5}\end{array}$ & $\begin{array}{c}\text { Sem disfagia } \\
\mathbf{n = 2}\end{array}$ & \multirow{2}{*}{ Valor de $\boldsymbol{p}^{* *}$} \\
\hline Cálcio $(\mathrm{mg})$ & $30,90-71,74$ & $37,42-50,64$ & $<0,05$ \\
Ferro $(\mathrm{mg})$ & $7,37-116,10$ & $7,37-29,62$ & $<0,05$ \\
Zinco $(\mathrm{mg})$ & $26,57-107,56$ & $35,47-85,39$ & $<0,05$ \\
Sódio* $\left.^{*} \mathrm{mg}\right)$ & $20,72-100,60$ & $21,44-65,87$ & $<0,05$ \\
Potássio $(\mathrm{mg})$ & $24,04-100,60$ & $45,92-62,17$ & $<0,05$ \\
Fósforo $(\mathrm{mg})$ & $12,02-38,02$ & $28,52-39,68$ & $<0,05$ \\
Vitamina C $(\mathrm{mg})$ & $75,03-129,23$ & $56,43-129,23$ & $<0,05$ \\
Vitamina A $(\square \mathrm{g})$ & $70,05-115,75$ & $112,22-154,89$ & $<0,05$ \\
Vitamina E $(\mathrm{mg})$ & $60,75-98,15$ & $64,39-89,04$ & $<0,05$ \\
\hline
\end{tabular}

* intrínsico

${ }^{* *}$ Teste do qui-quadrado exato de Fisher

\section{DISCUSSÃo}

Indivíduos portadores de MPS com a forma grave da doença evoluem para uma diarréia crônica, hidrocefalia, mau funcionamento das válvulas cardíacas, hipertensão e visceromegalias, levando a morte entre os dez e quinze anos de idade. Já a forma leve da doença traz menos sintomas, prolonga a vida do paciente até o início da fase adulta e possui como causa na maioria das mortes a obstrução das vias respiratórias ou falência cardíaca ${ }^{1,5,16,17}$. Assim, no grupo estudado, quase $50 \%$ dos indivíduos apresentava a forma grave da doença, e já fazia uso de anti-hipertensivos, apesar da pouca idade. Fato que evidencia nessa população uma evolução rápida e progressiva da MPS.

A MPS II é a única dos tipos de MPS com padrão de herança recessiva ligada ao cromossomo $\mathrm{X}$, acometendo principalmente pacientes do sexo masculino, comprovado na presente pesquisa, onde no estado de Alagoas todos os portadores de MPS II cadastrados no programa da UNCISAL são homens. Mulheres afetadas geralmente apresentam baixos níveis de atividade da 
iduronato - 2 - sulfatase (enzima deficiente na síndrome necessária para a degradação seqüencial dos GAGs dermatan e heparan sulfato) e um fenótipo clínico atenuado. As portadoras são assintomáticas, com exceção dos casos onde ocorre inativação do cromossomo $X^{1-5}$.

A literatura mostra que é comum o aumento de alguns órgãos, como coração, fígado e baço em portadores de MPS 1,3-5,9,16. Apesar disso, acredita-se que tal aspecto não tenha contribuído com o excesso de peso, e consequentemente a elevada frequência de sobrepeso e obesidade na população estudada. A baixa estatura para idade apresentada por esses indivíduos provavelmente colaborou para esse achado, o que pode sim explicar em partes a elevada freqüência de sobrepeso e obesidade, quando avaliados segundo o IMC, visto que quando os mesmos foram avaliados segundo o indicador $\mathrm{P} / \mathrm{I}$ apresentaram déficit leve, o que mostra que apresentam baixo peso segundo faixa etária. Tal fato vai de encontro ao que hoje é visto no Brasil e em todo o mundo, o processo de transição nutricional, com aumento na incidência de sobrepeso e obesidade ${ }^{18}$, o que reforça a hipótese da influência da doença de base sobre a depleção do estado nutricional ${ }^{7}$.

Vale salientar ainda que, o IMC não é um parâmetro totalmente fidedigno, pois não avalia separadamente a composição de reserva muscular e tecido adiposo ${ }^{19}$. Dentre estes indicadores utilizados a relação $A / l$ expressa o crescimento linear de crianças e corresponde ao dado que melhor representa o efeito cumulativo de situações adversas sobre o crescimento da criança. É considerado o índice mais sensível para aferir a qualidade de vida da população infantil. Apesar disso, a análise desse índice deve ser realizada com cautela, uma vez que existem crianças que são geneticamente (naturalmente) baixas, ou seja, o valor encontrado de uma baixa estatura para idade não é reflexo de condições patológicas ${ }^{20}$. Na presente pesquisa $85,71 \% \%$ dos portadores de MPS II, ou seja, 6 indivíduos, apresentaram déficit estatural segundo este indicador.

Pinto e colaboradores ${ }^{21}$ avaliaram prospectivamente, durante um período de 12 meses, onze pacientes brasileiros, com média de idade de 7,3 anos, portadores de MPS II provenientes do serviço de Genética Médica do Hospital de Clínicas de Porto Alegre, e encontraram resultados semelhantes ao nosso. Assim, após um ano de investigação, dos setes pacientes restantes na pesquisa (outros excluídos por óbito ou piora clínica), cinco $(71,42 \%)$ apresentaram déficit estatural segundo 0 indicador A/ I e sete deles (100\%) foram classificados com excesso de peso ou obesidade de acordo com $\mathrm{o}$ indicador $\mathrm{P} / \mathrm{A}$.
$\mathrm{Na}$ literatura pesquisada a maioria dos estudos feitos com portadores de MPS II estão dispostos na forma de relatos de casos e/ ou séries de caso que incluem pacientes com outras formas de MPS ${ }^{7}$. Estudos sobre a história natural das MPS II são escassos. Os pacientes costumam ser normais ao nascimento, e um curso progressivo é a regra, embora as etapas dessa progressão não estejam ainda bem delineadas. As manifestações clínicas mais freqüentemente encontradas são face grosseira, alterações esqueléticas, contraturas articulares, atraso do desenvolvimento neuropsicomotor, infecções recorrentes de vias aéreas superiores e inferiores, surdez, cardiopatia e baixa estatura ${ }^{5,6}$, como visto na presente pesquisa.

As necessidades nutricionais de cada indivíduo diferem entre si em função de fatores como: constituição genética, características morfológicas e fisiológicas, nível de atividade física e eficácia metabólica, além da presença de patologias. Portanto, considerando características e necessidades específicas, o cuidado nutricional deve manter um equilíbrio entre ingestão calórica, de nutrientes e necessidades nutricionais ${ }^{22}$. A baixa ingestão calórica nos indivíduos do presente estudo com MPS II em relação às suas necessidades energéticas diárias foi evidenciada a partir da média de consumo calórico diário contra as necessidades energéticas diárias. Além disso, foi observado um desequilíbrio na proporção e quantidade dos macronutrientes e isso foi dependente da faixa etária, com consumos adequados de proteínas e lipídios apenas nas faixas etárias de 4-8 anos e 9-18 anos, respectivamente.

É sabido que a MPS II é uma doença metabólica associada com o acúmulo de GAGs, por deficiência da enzima iduronato-2-sulfatase, promovendo acúmulo destas substâncias na células do corpo. A velocidade com que os sintomas aparecem, depende da velocidade de acúmulo dos GAGs. Os GAGs são açúcares produzidos normalmente no organismo. Assim, o açúcar ou outros alimentos fontes de carboidratos simples não irão afetar o acúmulo de $\mathrm{GAGs}^{1-3}$. Apesar de tal aspecto, é importante atentar para a qualidade deste carboidrato ofertado, visto que, o excesso de carboidratos simples e/ ou refinados apresenta uma relação direta com a obesidade, diabetes mellitus e a resistência à insulina, e consequentemente o surgimento de doenças cardiovasculares a longo prazo ${ }^{23}$.

Quanto à baixa ingestão de alguns micronutrientes pelos portadores de MPS II da presente pesquisa atenção deve ser dada ao baixo consumo de cálcio e potássio, visto que os mesmos em quantidades adequadas participam do controle da pressão arterial ${ }^{24}$ e como já relatado anteriormente, mais da metade dos indivíduos estudados tinham 
diagnóstico de hipertensão arterial sistêmica. Em contrapartida, o baixo consumo de sódio nesses indivíduos pode ser justificado pelo fato de apenas ter sido computado o sódio intrínsico, ou seja, aquele naturalmente presente nos alimentos, e não também o sódio na forma de cloreto de sódio, que seria o sal adicionado nas preparações alimentares, visto dificuldades dos acompanhantes dos menores em relatarem quantidades exatas do sal adicionado as preparações.

Estudos têm mostrado que o estresse oxidativo também ocorre nos Erros Inatos do Metabolismo, como na fenilcetonúria ${ }^{25}$, acidemias malônica e metilmalônica ${ }^{26}$, doença do xarope de bordo ${ }^{27}$, dentre outras. Em estudo realizado no Brasil ${ }^{28}$ foram verificados níveis aumentados de marcadores do estresse oxidativo, a exemplo de malondialdeído, grupamentos carbonilas plasmáticos, assim como da atividade da enzima catalase eritrocitária em pacientes com MPS II antes do tratamento de reposição enzimática específico para MPS quando comparados com o controle. É importante destacar que todos os portadores de MPS II do presente estudo não faziam uso de enzimas repositoras $e$, portanto, provavelmente estariam expostos ao estresse causado pela ação de espécies reativas. Assim, a terapia com antioxidantes seria uma estratégia vantajosa no controle dos danos celulares decorrentes da ação destas espécies ${ }^{29}$. Em nível dietético, foi observado após inquérito alimentar recordatório de 24 horas em cinco dias alternados, um baixo consumo dos antioxidantes zinco e vitamina $E$, em detrimento a um consumo adequado das vitaminas $A$ e $C$, que também apresentam importante papel na defesa contra espécies danosas ao organismo.

Outro achado importante na presente pesquisa foi o baixo consumo de ferro dietético por parte das crianças portadoras de MPS II. As anemias carenciais, em especial a decorrentes da deficiência de ferro, anemia ferropriva, ainda são no Brasil, e em especial na região nordeste, um grave problema de saúde pública em crianças e gestantes. Dentre os fatores relacionados com o surgimento de anemias está a dieta pobre em ferro de alto valor biológico, em especial os provenientes das carnes vermeIhas e vísceras ${ }^{30}$. Assim, os indivíduos em questão apresentam um potencial fator de risco para o surgimento de anemias carênciais e/ ou que justifique quadro de anemia nestes portadores de MPS II.

Os indivíduos com MPS II ao passar dos anos sofrem modificações na ingestão alimentar, em decorrências de uma série de fatores, como: dentição atrasada e deficiente, comprometendo a mastigação; alterações do trato gastrointestinal (constipação ou diarréia); dificuldades para se alimentar; perda visual, auditiva e na fala, levando na maioria das vezes a não aceitação do alimento oferecido $^{1,6}$. Logo essas e outras intercorrências afetam diretamente o estado nutricional destes pacientes, pois a ingestão dos mesmos torna-se desequilibrada.

Poucos são os estudos descritos na literatura no que se refere à avaliação fonoaudiológica em portadores de MPS, a maioria deles focaliza aspectos relacionados ao desenvolvimento da fala e da linguagem ${ }^{31}$. Turra \& Schwarts ${ }^{32}$, avaliando a motricidade oral em 78 pacientes com MPS atendidos no ambulatório de genética médica do hospital de clínicas de Porto Alegre, encontraram alterações em pelo menos um item de cada estrutura do sistema estomatognático ou função estomatognática, como exemplo alterações na arcada dentária, na língua, na mastigação e na deglutição, sendo está última alteração presente em $98,5 \%$ dos portadores de MPS. Na avaliação funcional da deglutição no presente estudo houve presença de disfagia em mais de $70 \%$ dos portadores de MPS II avaliados. É importante salientar que os indivíduos que apresentam atraso ou falha no reflexo do mecanismo de deglutição, tornam-se incapazes de formar o bolo alimentar e propulsionar o alimento para o esôfago.

A variabilidade da dieta do indivíduo está sujeita à variação real dos alimentos consumidos, influenciada pela heterogeneidade da dieta e pelas preferências. Além disso, sofre influência da sazonalidade, dias da semana, sequência da aplicação do inquérito dietético, etc. ${ }^{33}$, o que requer, quando se opta pelo recordatório de 24horas, um maior número de dias de investigação possível. Nesse aspecto apresenta a fórmula para cálculo do número de dias necessários para estimar a ingestão real de um nutriente a depender do grau de acurácia e do coeficiente de variação intraindividual do nutriente em questão. No entanto, esta metodologia nem sempre é de fácil aplicação. Porém, esta orientação aponta para a importância de conhecer o coeficiente de variabilidade de ingestão dos nutrientes num determinado espaço de tempo. Como visto, os indivíduos estudados apresentaram uma elevada variação de consumo de nutrientes na dieta. Isso reflete, por muitas vezes, uma baixa condição sócio-econômica existente, pois muitos sobrevivem apenas da ajuda do governo, ou a própria condição de disfagia, visto que a variação de consumo alimentar foi mais evidente no grupo com essa manifestação clínica.

Os aspectos acima descritos e as dificuldades na alimentação destes indivíduos, além das alterações dentárias comprometem o padrão de mastigação e deglutição e podem acarretar em privação nutricional ${ }^{6}$. Estes fatores que, direta ou indiretamente resultam em ingestão alimentar reduzida 
comprometendo o estado nutricional dos pacientes, o que contribui para o aumento da incidência de complicações secundárias à patologia de base.

Assim, a consistência pastosa é considerada a mais indicada para crianças com disfunção motora oral, por ser de mais fácil manipulação, reduzir tosse e aspiração, além de facilitar a alimentação da criança ${ }^{34}$. Estudo relata que em pacientes com MPS II foi evidenciado uma significante alteração na função oral e dificuldade com os alimentos sólidos repercutindo no estado nutricional desses pacientes ${ }^{6,8}$.

Conforme o grau de disfagia diagnosticado e as limitações do paciente, o manejo nutricional objetiva facilitar a deglutição tornando-a segura e independente, prevenindo o risco de aspiração e engasgos, manter e/ou recuperar o estado nutricional do paciente, considerando fatores para o planejamento dietético como: consistência, firmeza, viscosidade e densidade dos alimentos ${ }^{35}$.

\section{CONCLUSÃO}

Foi observada no presente estudo uma elevada freqüência de disfagia nos portadores de MPS II estudados, inclusive em todos aqueles com a forma severa da doença, e isso foi associado ao baixo consumo alimentar calórico e desequilíbrio na proporção e quantidade de macronutrientes e de parte dos micronutrientes.

Devido ao grande risco de carências nutricionais e mediante resultados expostos, há necessidade de um acompanhamento multidisciplinar. A intervenção nutricional conjunta com o acompanhamento fonoaudiológico, se torna fundamental na tentativa de minimizar os sintomas apresentados, determinar a via alimentar segura, diminuir o risco de aspiração e otimizar a ingestão alimentar adequada, repercutindo assim positivamente na qualidade de vida destes indivíduos.

\section{ABSTRACT}

Purpose: this study aimed to relate the degree of dysphagia and food consumption of individuals with mucopolysaccharidosis II (MPS II). Method: it was included individuals with MPS II of the genetics department at the State University of Alagoas and excluded those with other types of mucopolysaccharidosis and in use of alternative way of supply. There were performed anthropometric, dietetic, speech therapy for dysphagia,clinical evaluation and otorrinolarigologic videoendoscopy swallowing. Results: there were studied 07 individuals, male, between 5 and 14 years old: from them, more than $50 \%$ were taking anti-hypertensive and $42.8 \%$ had the severe form of disease. Six had serious deficit height / age and over $70 \%$ were obese according to Body Mass Index (BMI). Dysphagia was observed in five subjects, with daily average caloric intake $920.15 \pm 244.09$ calories, against $1264.94 \pm 106.85$ calories for those without such changes, with significantly greater intraindividual variation in the group of patients with dysphagia $(p<0.05)$. In addition, individuals without dysphagia had higher food intake of carbohydrates, proteins and lipids. As for micronutrients, except average daily intake of iron and vitamin E, all other assessed had higher average daily consumption in the group without dysphagia $(p<0.05)$. Conclusion: it was observed a high frequency of dysphagia in the studied patients with MPS II, and this was associated with low caloric food consumption and imbalance in the proportion and amount of macronutrients and part of micronutrients.

KEYWORDS: Deglutition Disorders; Mucopolysaccharidosis II; Diet; Food Consumption

\section{REFERÊNCIAS}

1. Schwartz IVD, Ribeiro MG, Mota JG, Toralles MBP, Correia P, Horovitz D, et al. A clinical study of 77 patients with mucopolysaccharidosis type II. Acta Paediatrica. 2007; 96, 63-70.

2. Cho YS, KIM JH, Chung SC, Chang AS, JIN DK. Otologic Manifestation of Hunter Syndrome and their relationship eith speech development. Audiol Neurotl, 2008; 13: 206-12.

3. Martins AM, Dualibi AP, Norato D, Takata ET, Santos ES, Valadares ER, et al. Guidelines for management of mucopolysaccharidosis type I. The Journal of Pediatrics. 2009; 155 (4): 31-45.

4. Garcia AR, Pan JC, Lamsa M, Muenzer J. The characterization of a murine model of 
mucopolysaccharidosis II (Hunter's syndrome). J Inherit Metab Dis. 2007; 30:924-34.

5. Wraith JE, Scarpa M, Beck M, Bodamer OA, Meirleir L, Guffon N, et al. Mucopolysaccharidoses type II (Hunter syndrome): a clinical review and recommendations for treatment in the era of enzyme replacement therapy. Eur J Pediatri. 2008; 167: 267-77.

6. Muenzer J, Wraith JE, Clarke LA. Mucopolysaccharidosis I: management and treatment guidelines. Pediatrics. 2009; 123 (1): 19-29.

7. Logemann JA. Oropharyngeal dysphagia and nutritional management. Current Opinion in Clinical Nutrition and Metabolic Care. 2007; 10: 611-4.

8. Ferreira ACRG, Silva RCF, Azevedo CLC, Dizeu LTB, Ferraciu CCS, Silva EHA, et al. Perfil do Sistema estomatognático na mucopolissacaridose tipo II em estágio avançado: estudo de caso. Suplemento especial da Revista da sociedade brasileira de Fonoaudiologia, 2006; Suppl AO:173.

9. Martins AN, Micheletti C, Tavares FM, Santos IMG, Oliveira RB, Guedes ZCF. Atuação fonoaudiológica junto aos portadores de doenças com erros inatos do metabolismo. In Hernandes AN, Marchesan I. Atuação fonoaudiológica no ambiente hospitalar, 1a ed. Rio de Janeiro: Revinter; 2001. p.127-42.

10. World Health Organization. Multicentre Growth Reference Study Group. WHO Child Growth Standards: Length/height-for-age, weight-for-age, weight-for-length, weight-for-height and body mass index-for-age: Methods and development. Geneva: World Health Organization, 2006; 312 p.

11. Fisberg RM, Marchioni DML, Colucci ACA. Avaliação do consumo alimentar e da ingestão de nutrientes na prática clínica. Arq Bras Endocrinol Metab. 2009; 53 (5): 617-24.

12. Frazão YS. Disfagia na Paralisia Cerebral. In: Léslie Piccolotto Ferreira; Débora M. Befi-Lopes; Suelly Cecília Olivan Limongi. (Org.). Tratado de Fonoaudiologia. São Paulo: Roca Ltda; 2004, p. 370-85.

13. Macedo Filho ED. Avaliação videoendoscópica da deglutição (nasofaringoscopia da deglutição). In: Milton Costa e Luiz de Paula Castro. Tópicos em deglutição e disfagia. Rio de Janeiro: Medsi, 2003, p. 285-97.

14. IOM (Institute of Medicine). Dietary Reference Intakes: Applications in Dietary Planning. Waschington, DC: National Academies Press, 2003, 2001. http://www.nap.edu/catalog/10609.html 15. Institute of Medicine (IOM). Dietary reference intakes for energy, carbohydrate, fiber, fat, fatty acids, cholesterol, protein, and amino acids (macronutrients). Washington, DC: National Academy Press, 2002/ 2005.
16. Jeong Han-Sin, Cho Do-Yeon, Ahn Kang MO, Jin Dong-Kyu. Complicatons of tracheotomy in patients with mucopolysaccharidosis II (Hunter's syndrome). International Journal of Pediatric Otorhinolaryngology. 2006; 70: 1765-9.

17. Martin et al. Recognition and Diagnosis of mucopolysaccharidosis II (Hunter Syndrome). Official Journal of the American Academy of Pediatrics. 2008; 121:377-86.

18. Bradford NF. Overweight and Obesity in Children and Adolescents. Primary Care: Clinics in Office Practice. 2009; 36: 319-39.

19. Cole TJ, Bellizi MC, Flegal KM, Nicholls D, Jackson AA. Body mass índex cut offs to define thinness in children and adolescents: international survey. BMJ. 2007; 335 (7612): 166-73.

20. Soares NT. Um novo referencial antropométrico de crescimento: significados e implicações. Rev. Nutr. Campinas. 2003; 16 (1): 93-104.

21. Pinto LLC, Schwartz IVD, Puga ACS, Vieira TA, Munoz MVR, Giugliani R, et al. Avaliação prospectiva de 11 pacientes brasileiros com mucopolissacaridose II. J. Pediatr. 2006; 82 (4): 273-8.

22. Araújo CL, Albernaz E, Tomasis E, Victoria CG. Food and Nutrition Bulletin. 2004; 25 (1): 53-60.

23. Ribeiro RQC, Lotufo PA, Lamounier JA, Oliveira RG, Soares JF, Botter DA. Fatores adicionais de risco cardiovascular associados ao excesso de peso em crianças e adolescentes. O estudo do coração de Belo Horizonte. Arquivos Brasileiros de Cardiologia. 2006; 80 (6): 408-18.

24. Hackman DG, Khan NA, Hemmelgarn BR, Rabkin SW, Touyz RM, Campbell NRC, et al. The 2010 Canadian Hypertension Education Program Recommendations for the management of hypertension: part 2. Canadian Journal of Cardiology. 2010; 26 (5): 249-58.

25. Sitta A, Barschak AG, Deon M, De Mari JF, Barden AT, Vanzin CS, et al. L- carnitine blood levels and oxidative stress in treated phenylketonuric patients. Cell Mol Neurobiol. 2009; 2: 211-8.

26. Ribas GS, Manfredini V, De Marco MG, Vieira RB, Wayhs CY, Vanzin CS, et al. Prevention by L-carnitine of DNA damage induced by propionic and L-methylmalonic acids in human peripheral leukocytes in vitro. Mutat Res. 2010; 702: 123-8.

27. Barschark AG, Sitta A, Deon M, Busanello EN, Coelho DM, Cipriane F, et al. Amino acids levels ad lipid peroxidation in maple syrup disease patients. Clin Biochem. 2009; 42 (6): 462-6.

28. Filippon L, Vanzin CS, Biancini GB, Pereira IN, Manfredini V, Sitta A, et al. Oxidative stress in patients with mucopolysaccharidosis type II before and during enzyme replacement therapy. Molecular Genetics and Metabolism. 2011; 103 (2): 121-7. 
29. Vasconcelos SML, Silva MAM, Goulart MOF. Pró-antioxidantes e antioxidants de baixo peso molecular oriundos da dieta: estrutura e função. Nutrire Rev. Soc. Bras. Alim. Nutr. 2006; 31 (3): 95-118.

30. Jordão RE, Bernardi JLD, Filho AAB. Prevalência de anemia ferropriva no Brasil: uma revisão sistemática. Rev Paul Pediatr. 2009; 27 (1): 90-8.

31. Cho YS, Kim JH, Kim TW, Chung SC, Chang AS, Jin DK. Otologic manifestations of Hunter syndrome and their relationship with speech development. Audiol Neurootol. 2008; 13: 206-12.
32. TurraGS, Schwarts IVD. Avaliação damotricidade oral em pacientes com mucopolissacaridose: um estudo transversal. Jornal de Pediatria. 2009; 85 (3): 254-60.

33. Slater B, Marchioni DL, Fisberg RM. Estimando a prevalência da ingestão inadequada de nutrientes. Rev. Saúde pública 2004; 38(4): 599-605.

34. Vivone GP, Tavares MMM, Bartolomeu RS, Nemr K, Chiappetta ALML. Análise da consistência alimentar e tempo de deglutição em crianças com paralisia espástica. Revista Cefac. 2007; 9: 504-11. 35. Gonzalés LL, EnterríaPG. Tratamiento nutricional de La disfagia orofaríngea. Endocrinología y Nutricíon 2006; 53 (5): 309-14.

Endereço para correspondência:

Ana Carolina Rocha Gomes Ferreira Avenida José Airton Godim Lamenha, Lote 10, Quadra D, bairro São Jorge Maceió, Alagoas, Brasil CEP: 57044-098

E-mail: acr_fono@yahoo.com.br 